\title{
Prediction of Rail Contact Fatigue on Crossings Using Image Processing and Machine Learning Methods
}

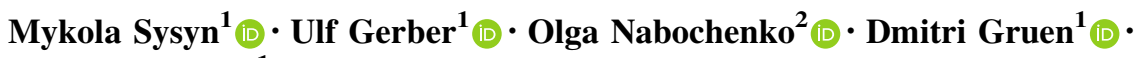 \\ Franziska Kluge ${ }^{1}$ (D)
}

Received: 4 December 2018/Revised: 16 May 2019/Accepted: 24 May 2019/Published online: 4 June 2019

(C) The Author(s) 2019

\begin{abstract}
In this paper, an application of computer vision and machine learning algorithms for common crossing frog diagnostics is presented. The rolling surface fatigue of frogs along the crossing lifecycle is analysed. The research is based on information from high-resolution optical images of the frog rolling surface and images from magnetic particle inspection. Image processing methods are used to pre-process the images and to detect the feature set that corresponds to objects similar to surface cracks. Machine learning methods are used for the analysis of crack images from the beginning to the end of the crossing lifecycle. Statistically significant crack features and their combinations that depict the surface fatigue state are found. The research result consists of the early prediction of rail contact fatigue.
\end{abstract}

Mykola Sysyn

mykola.sysyn@tu-dresden.de

Ulf Gerber

ulf.gerber@tu-dresden.de

Olga Nabochenko

olganabochenko@gmail.com

Dmitri Gruen

dimitri.gruen@tu-dresden.de

Franziska Kluge

franziska.kluge1@mailbox.tu-dresden.de

1 Institute of Railway Systems and Public Transport, Technical University of Dresden, Dresden, Germany

2 Dnipropetrovsk National University of Railway Transport, Dnipro, Ukraine

Communicated by Peng Xu
Keywords Railway turnout - Common crossing - Image processing $\cdot$ Rolling contact fatigue $\cdot$ Machine learning · Feature detection and selection

\section{Introduction}

Railway turnouts are high-asset and maintenance-intensive parts of the railway superstructure. They are a limiting factor in a reliable and cost-effective railway infrastructure due to their short lifecycles and the difficulties in predicting the remaining useful life of turnout elements [1]. Despite significant advances in the application of various automated measurement and diagnostic systems $[2,3]$ in the course of railway digitalization, the predictability of the crossing lifetime is low. The reasons include not only a large uncertainty of inertial measurements due to many random influences [4] but also the complexity of degradation processes [5].

Many different systems [6] are used for the inspection of common crossing rolling surfaces: profile, surface scan and video inspection, microstructure imaging, eddy current and ultrasound, vehicle-based and track-based inertial measurements (Fig. 1). However, none of the systems can yet replace the conventional inspection method, which includes expert judgement based on visual estimation and acoustic perception of train impacts.

Profilometer methods deliver information on the longitudinal wheel trajectory and cross section of common crossings, which is simple to interpret. Their main drawback is that the measurement takes place in an unloaded state. Surface scanning methods show similar limitations, while at the same time, the additional measurement information can also demand additional interpretation. The German Railways (DB AG) tested laser surface scanning 
Fig. 1 Common crossing inspection methods

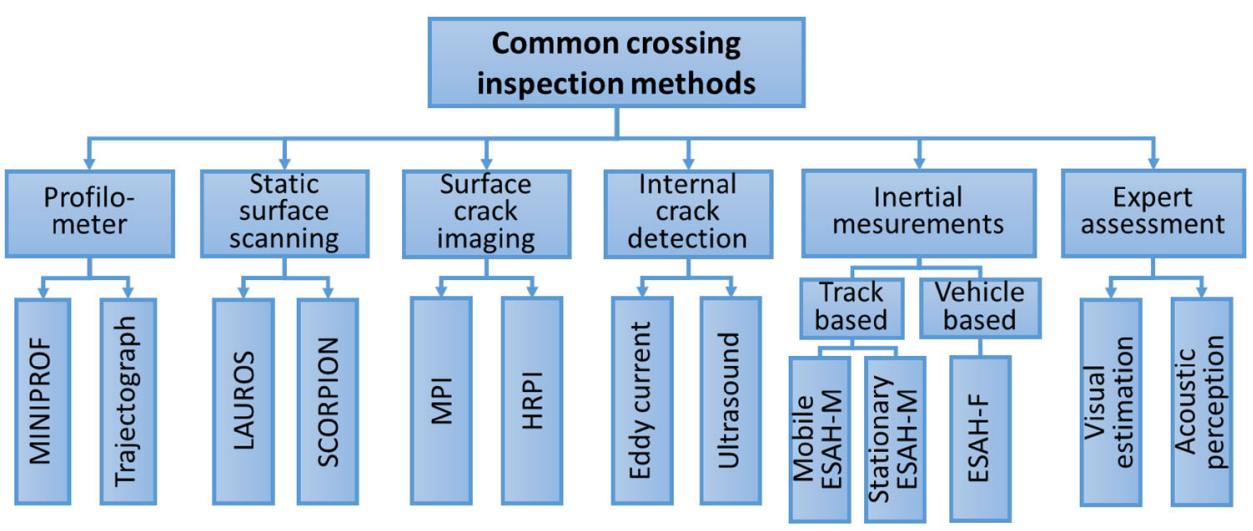

methods for common crossings at Scorpion and Lauros (Fig. 2). Both methods depict the wear state of the rolling surface, and the additional instantaneous high-resolution imaging is considered.

The conventional methods for rail fatigue assessment are eddy current and ultrasonic methods [9]. Both methods can primarily be considered as fault-detection methods that are not able to detect crack origination. Vehicle-based inertial measurement, like the axle box ESAH-F measurement system [3], which is installed on regular trains, allows inspecting a big number of turnouts with low expenses. The application of the system is limited to the detection of existing faults or wear, without prediction of common surface damages.

Magnetic particle inspection (MPI) is a reliable method to detect surface features. However, it is a very time-consuming inspection method with a low degree of automatization. High-resolution photo inspection (HRPI) would be a promising alternative to MPI. It enables highly automatized application in measuring cars as well as in easy practical inspection with mobile devices. Figures 3 and 4 show MPI and HRPI images of a frog nose rolling surface during its normal operation at $33 \mathrm{Mt}$ and after rolling surface fatigue damages at $52 \mathrm{Mt}$. The MPI image clearly demonstrates a different MPI crack image pattern at $33 \mathrm{Mt}$ in zones corresponding to the fatigue fault at $52 \mathrm{Mt}$. Thus, it can be considered a characteristic pattern of the future fatigue zones. However, it is difficult to assess the remaining useful life of the rolling surface, since the patterns can appear long before the visible cracks.

The HRPI images, different to those of MPI, from the first sight give no indication about the imminent surface fault. However, experienced experts can clearly detect it and consider that the HRPI images are not less informative than the MPI images. The main problem of high-resolution photo inspection is the difficulty of automatic crack recognition in the early phase of their emergence. It is therefore necessary to develop image processing methods that are able to transform the HRPI images to images of a form that corresponds to MPI images without substantial loss of information.

Image processing and machine learning methods are successfully used in civil and transportation engineering. A dimensionality reduction method, like principal component analysis, is used in the study [10] to assess the condition of bridges using the data collected during visual inspections. Fatigue fracture diagnostics of building structure elements, including microhardness measurements and statistical processing, are used in [11]. An evaluation of railway ballast consolidation with discriminant and cluster analysis is proposed in [12]. A histogram-based image segmentation method, which is proposed in [13], is a promising technique for pre-processing HRPI images of the rail rolling surface. Raster image processing methods are used in [14] for the extraction of objects of interest from point clouds and their automatic classification.
Fig. 2 Surface scanning methods for common crossings. (left, Scorpion [7]; right, Lauros [8])

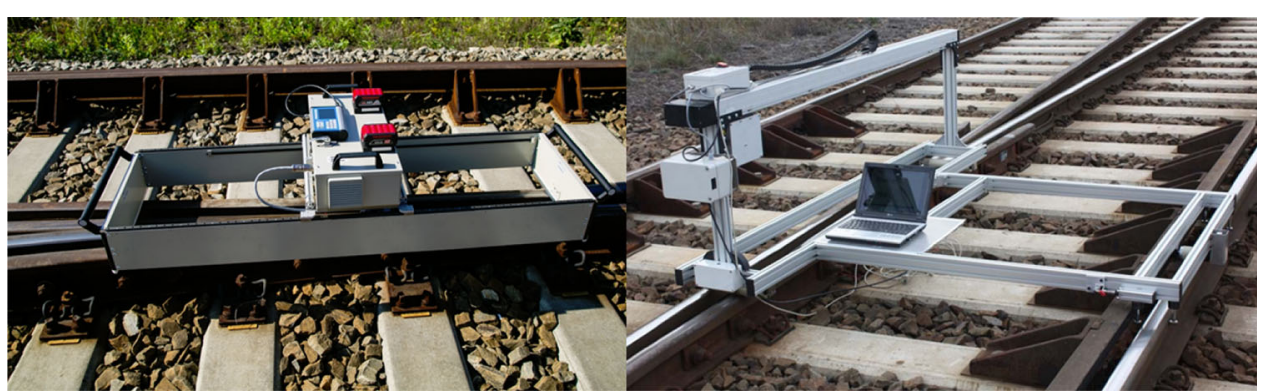




\section{I}

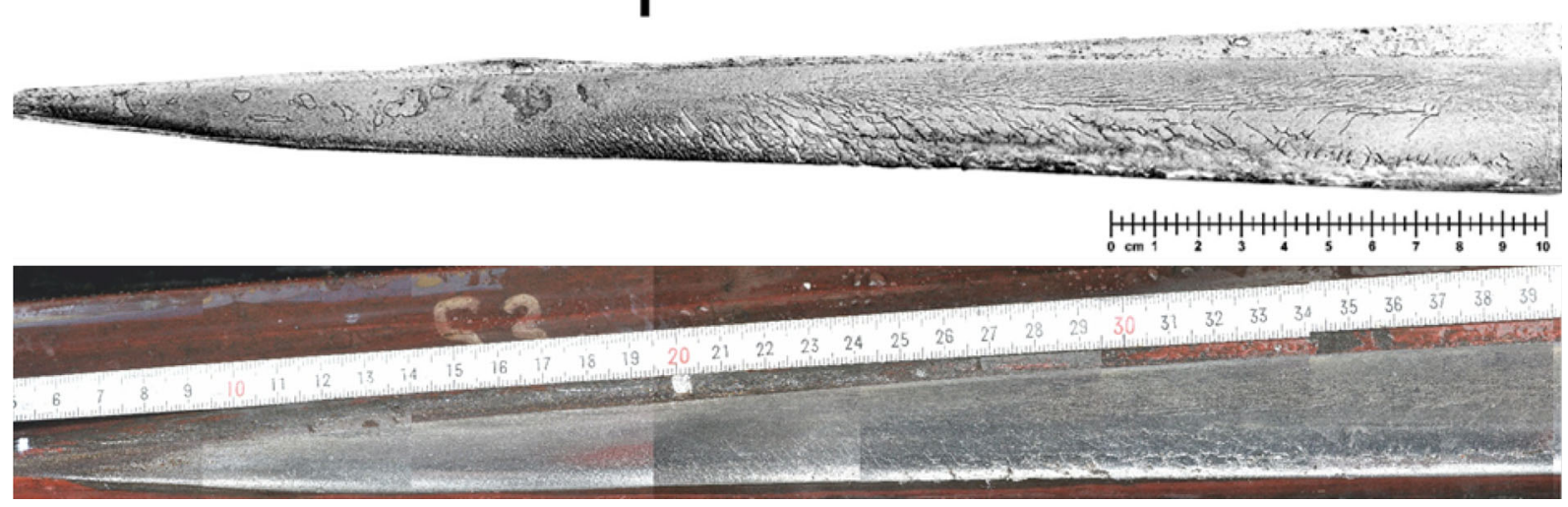

Fig. 3 MPI (above) and HRPI (below) images of the rolling surface on a frog nose after $33 \mathrm{Mt}$

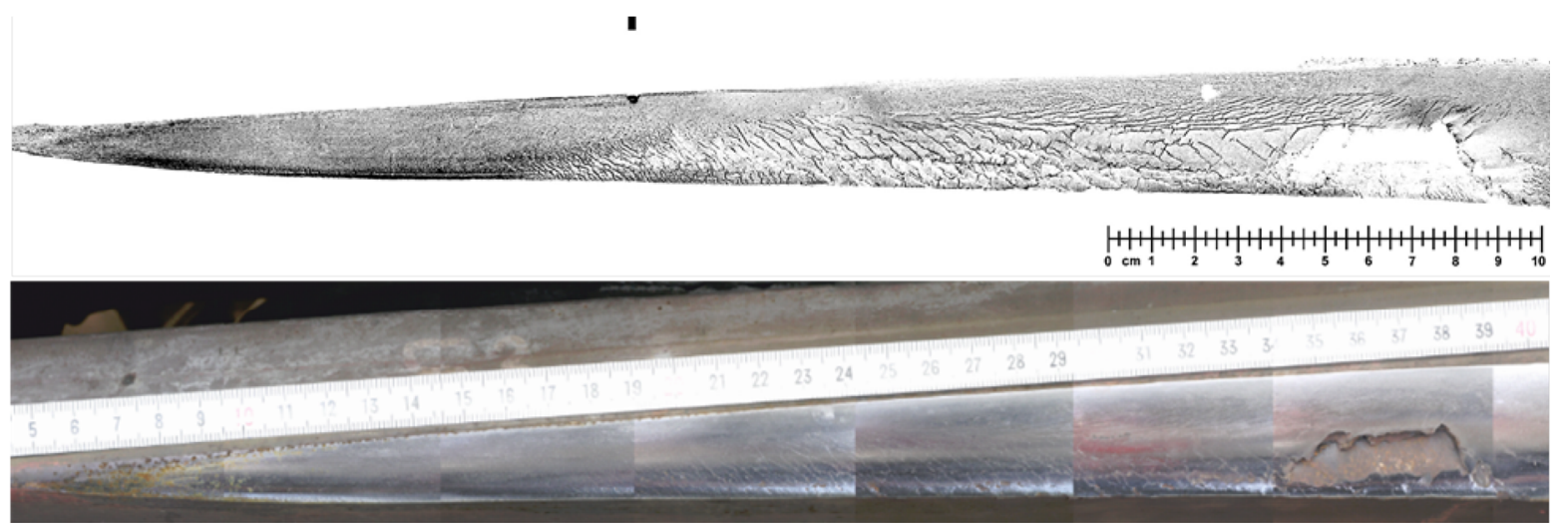

Fig. 4 MPI (above) and HRPI (below) images of the rolling surface on a frog nose after $52 \mathrm{Mt}$

Deep learning pertaining to models is applied in [15] to improve the automatized processing of crack images in concrete structures. The approach of machine learning predictive detection that is introduced in [16] could be used to improve the predictive detection of rolling surface degradation. A rail surface inspection method using deep learning and image processing is proposed in the study [17]. Paper [18] shows an approach for semi-supervised rail defect detection with the aim of improving the performance of squat detection. The authors of the study [19] propose an improvement of head surface defect detectionbased video inspection and image processing methods. An early detection of common crossing rolling contact faults with vehicle-based inertial measurements and machine learning methods is studied in [20]. A mechanical modelling of short-term dynamic interaction and long-term settlements of common crossing is presented in [21]. The modelling results are compared with those of the on-board inertial measurements. The results can be used to improve common crossing lifecycle prediction.

The general feature of the reviewed studies of rolling surface diagnostics is that most of them consider fault detection in the late state of development without the prediction of their following growth. The aims of the present research are the objectification and automatization of the conventional human visual inspections, as well as discovering the possibilities for early prediction of rail contact fatigue in common crossings.

\section{Approach Description in General}

The main part of the paper consists of the solution of the following problem: detecting the feature changes in the crack images that are statistically related to the frog lifetime. The problem is solved by using image processing and statistical image analysis. A workflow diagram of the present research is shown in Fig. 5.

The image processing and statistical image analysis is based on data collected from one frog during its lifecycle. The information consists of 5 MPI images of the frog's rolling surface after 13, 22, 33, 43 and 52 Mt. The image processing and the following statistical analysis are based on those MPI images. Additional information sources are the HRPI images after 13, 33 and $52 \mathrm{Mt}$, which are used as 


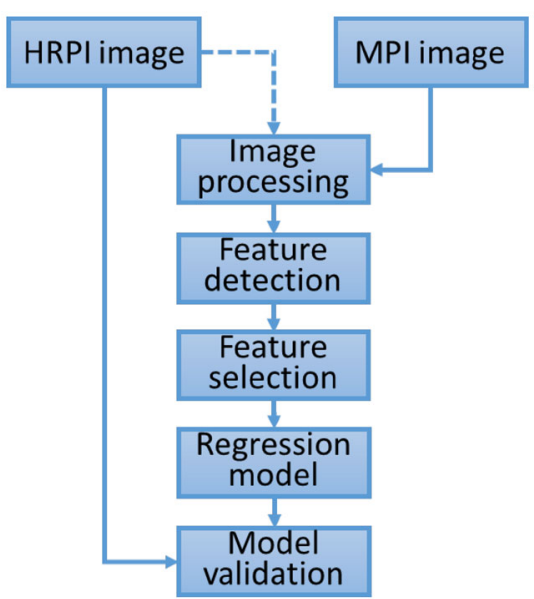

Fig. 5 Workflow diagram of image processing and statistical image analysis

independent information for the validation of the statistical model.

\section{Image Pre-processing and Feature Detection}

To prepare the images for the feature detection, they are initially improved with morphological image enhancement techniques, which increase the signal-to-noise ratio of images. The subsequent dilation and erosion operations are used to remove small objects from an image and to smooth the border of large objects [22].

The extraction of the meaningful information from the improved images is carried out by using image analysis. During the analysis, the independent image objects are detected and their properties are measured. The following table shows all used properties, providing a description of the shape measurements and the used abbreviations (Table 1).
The MPI images after the enhancement techniques still show a big number of objects that are evidently not related to crack images. The line-shaped objects are extracted as a pre-processing step, using two simple conditions: the $\mathrm{Ar}$ feature is limited within a minimal, and a maximal value and the $P A R$ feature is assumed to be more than 0.5. Figure 6 shows the MPI image, filtered using the two conditions, with object colours corresponding to their values of the $P A R$ feature. A remarkable observation is that the cracks with a big $P A R$ feature, mostly belonging to small cracks, are randomly distributed over the rolling surface. Obviously, the PAR feature itself could not be considered as a characteristic for the rolling surface state estimation.

\section{Preliminary Statistical Analysis}

The overall statistic of crack objects from 5 images during the frog's lifecycle contains 940 observations with 12 predictors and 5 classes of response variables. A preliminary statistical analysis is carried out to describe the general properties of the statistics. The data are of different dimensions, e.g. linear, angular or area measures, which causes some difficulties while comparing different features. To simplify the problem, all the data are reduced to undimensional values or normalized. In their normalized state the mean values of the features equal 1 .

The second particularity of the data is a very big asymmetrical variability. Figure 7 with boxplots for all 12 features shows that most of them have a variability inside the upper and lower quartiles, which is comparable with their median value. The Orn (Angle between the $\mathrm{x}$-axis and the major axis of the ellipse) feature, that corresponds to the crack orientation, has much bigger variability due to positive and negative values.
Table 1 The measured properties and their abbreviations

\begin{tabular}{ll}
\hline Abbr. & Description \\
\hline Ar & Area or number of pixels in the region \\
MajAxLn & Length in pixels of the major axis of the ellipse that approximates the region \\
MinAxLn & Length in pixels of the minor axis of the ellipse that approximates the region \\
Orn & Angle between the x-axis and the major axis of the ellipse \\
ConvAr & Number of pixels in convex image, with all pixels within the hull filled \\
FilAr & Area or number of pixels in the region without internal regions \\
EN & Number of objects in the region minus the number of holes in those objects \\
EqDm & Diameter of a circle with the same area as the region \\
Sol & Proportion of the pixels in the convex hull that are also in the region \\
$E x t$ & Ratio of pixels in the region to pixels in the total bounding box \\
Per & Perimeter or distance in pixels around the boundary of the region \\
PAR & Perimeter to area relation
\end{tabular}




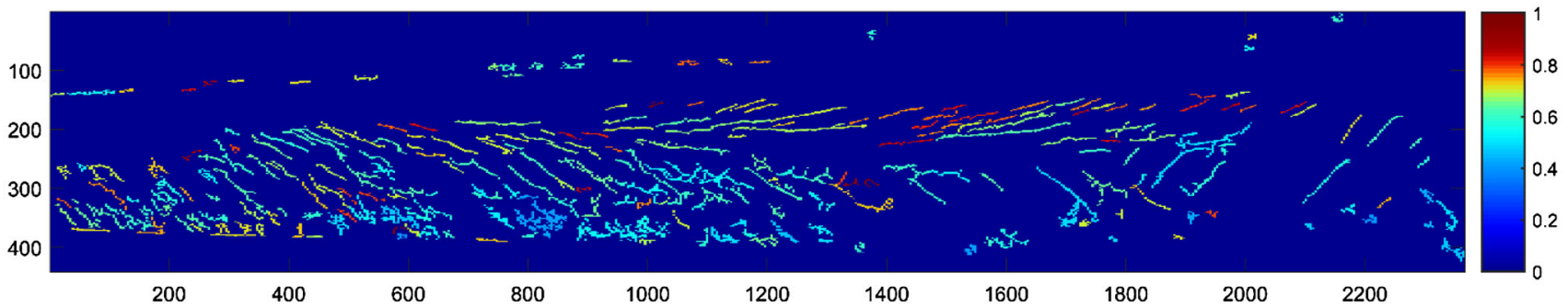

Fig. 6 Image objects with their $P A R$ features (43 Mt)

Fig. 7 Box plots of features for image objects
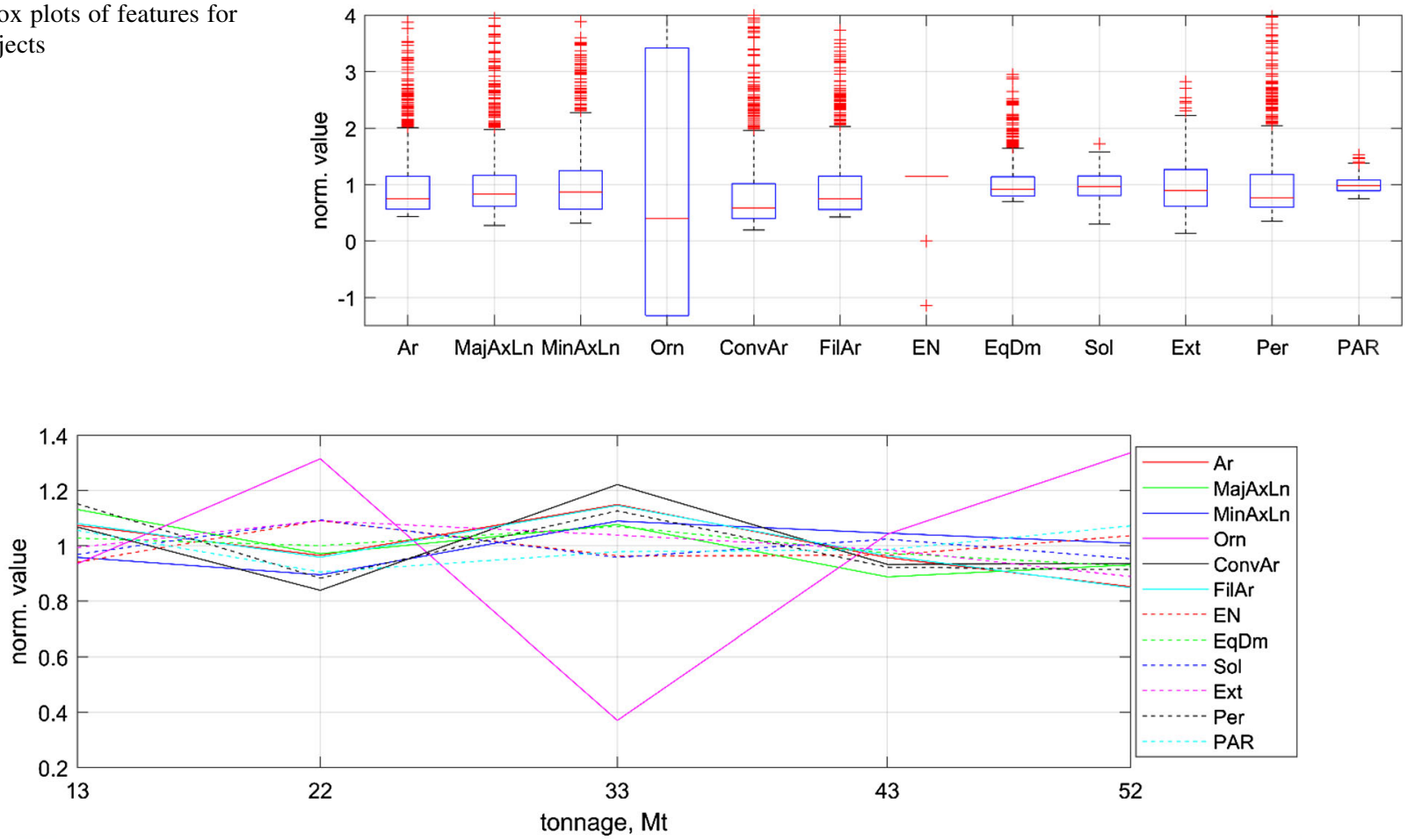

Fig. 8 The trend of the feature mean values

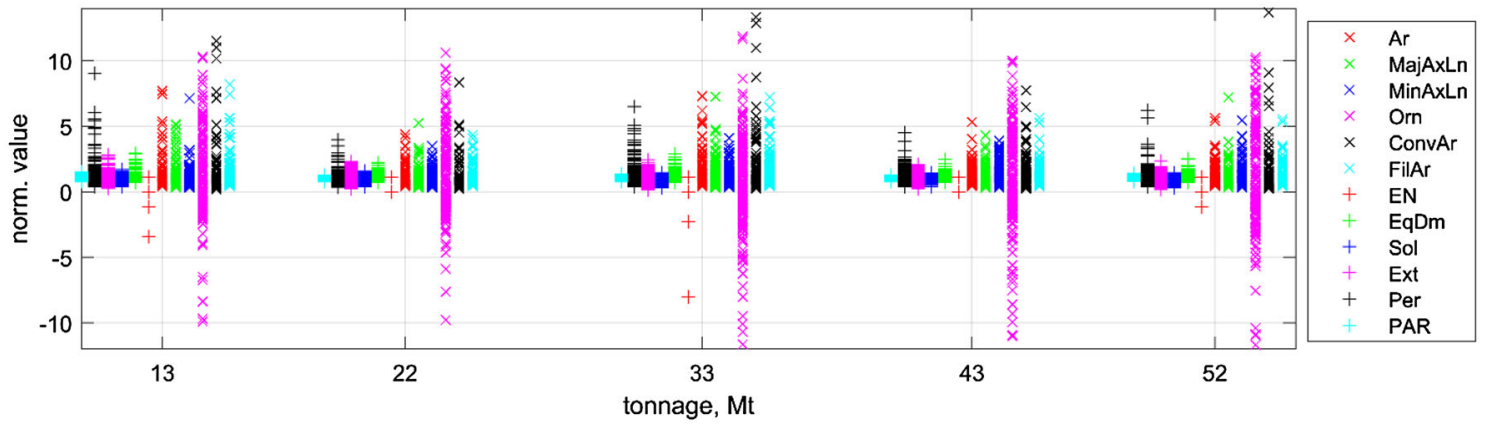

Fig. 9 Normalised feature values of image objects

The mean values of the features depending on the tonnage are depicted in Fig. 8. The trend of the mean values is rather random and ambivalent.

Figure 9 shows the variability of the data along the frog's lifecycle. Many features show very similar values, which means that they do not carry new information and therefore could be supposed redundant. The diagrams show no evident relation of the measured features to the frog's lifetime. 


\section{Principal Component Analysis and Feature Selection}

To reveal the relations between the features that are still hidden in the Figs. 8 and 9, the principal component analysis (PCA) is used. PCA is mostly used as a means of exploratory data analysis and for making predictive models $[23,24]$. It helps to reveal the internal structure of the data in a way that best explains the variance in the data. The sense of the PCA consists of replacing a group of variables with a single new variable, called principal components. Each principal component is a linear combination of the original variables. All the principal components are orthogonal to each other, so there is no redundant information [25]:

$z_{i 1}=\varphi_{11} x_{i 1}+\varphi_{21} x_{i 2}+\cdots+\varphi_{p 1} x_{i p}$

here: $z_{i 1}$ are the scores, and $\varphi_{11}, \ldots, \varphi_{p 1}$ is the loadings of the first principal component.

The following pareto diagram (Fig. 10) shows the first 6 components that explain $95 \%$ of the total variance. The first component explains a bigger share of the variance than all other components together.

However, Fig. 10 cannot explain the reason for the variance. The particularity of PCA is that it is an unsupervised approach, since it involves only a set of features. To find out what component corresponds to the response variable, i.e. the frog's lifetime, the scatter plot of data in the coordinates of the first two components is built (Fig. 11). Together with this, a biplot is depicted that allows visualization of the magnitude and sign of each feature's contribution to the first two components. The first principal component, on the horizontal axis, is strongly influenced by the features Ar, Per, ConvAr, EqDm and less strong by MinAxLn, MajAxLn, all of them in a positive direction. The second principal component, on the vertical axis, has positive coefficients for the $P A R$ end $E N$ features and negative for Ext, Sol and Orn. The different lifetime is depicted using different colours from red to blue. Apparently, more blue points with a tonnage of 43 and $52 \mathrm{Mt}$ are concentrated in the positive direction of the second component. Although the first component has four times bigger weight than the second, it has no relation to the frog's lifetime.

The PCA shows that many of the 12 features are redundant. To select the meaningful features for the first two components, their weights are plotted for each feature (Fig. 12). The Pcl has the following four most meaningful features and weights: $\operatorname{Ar}(0.21), \operatorname{ConvAr}(0.94), \operatorname{FilAr}(0.22)$ and $\operatorname{Per}(0.13)$. The $P c 2$ has the following: $\operatorname{Ar}(0.60)$, MajAxLn(0.11), ConvAr(0.33), FilAr(0.61) and Per(0.36).

Both $P c 1$ and $P c 2$ are influenced by a similar range of features: Ar, MajAxLn, FilAr and Per. The only difference between $P c 1$ and $P c 2$ consists in the feature ConvAr, which for $P c 2$, has a negative value. The relation of the mean values of $P c 1$ and $P c 2$ with the selected features to the frog's lifetime is shown in Fig. 13. The shown $P c$ values are not normalised, but the beginning values are reduced to 0 for a convenient visualisation. Figure 13 shows the monotonous relation of $P c 2$ to the frog's lifetime. Although $P c 1$ has a much bigger weight than $P c 2$, it cannot provide an unambiguous relation to the lifetime. The variance of the $P c 2$ value could be explained by differences in the image acquisition due to the MPI technique, such as contrast, brightness of illumination, etc.

The main advantage of PCA is that it provides an easy interpretable indicator that demonstrates systematic variation versus crossing lifetime and takes into account the
Fig. 10 The pareto diagram of total variance explained with PCA components

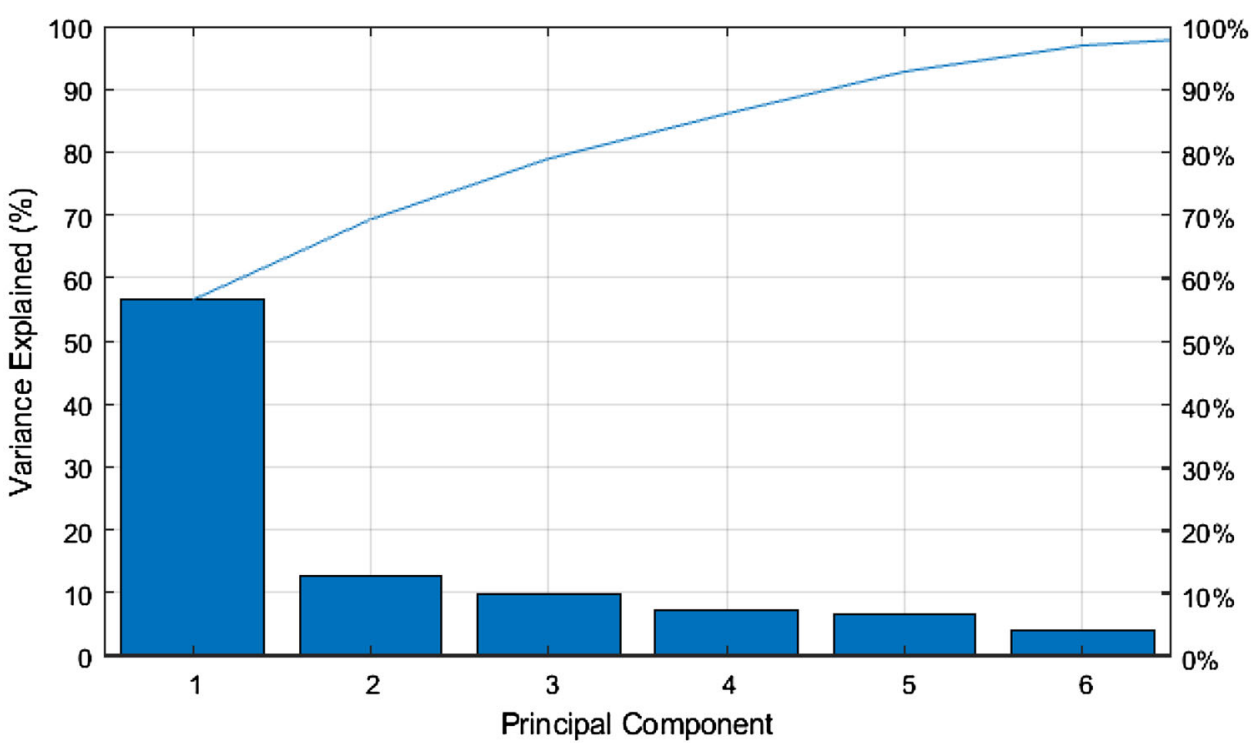


Fig. 11 The first two principal components and feature vectors

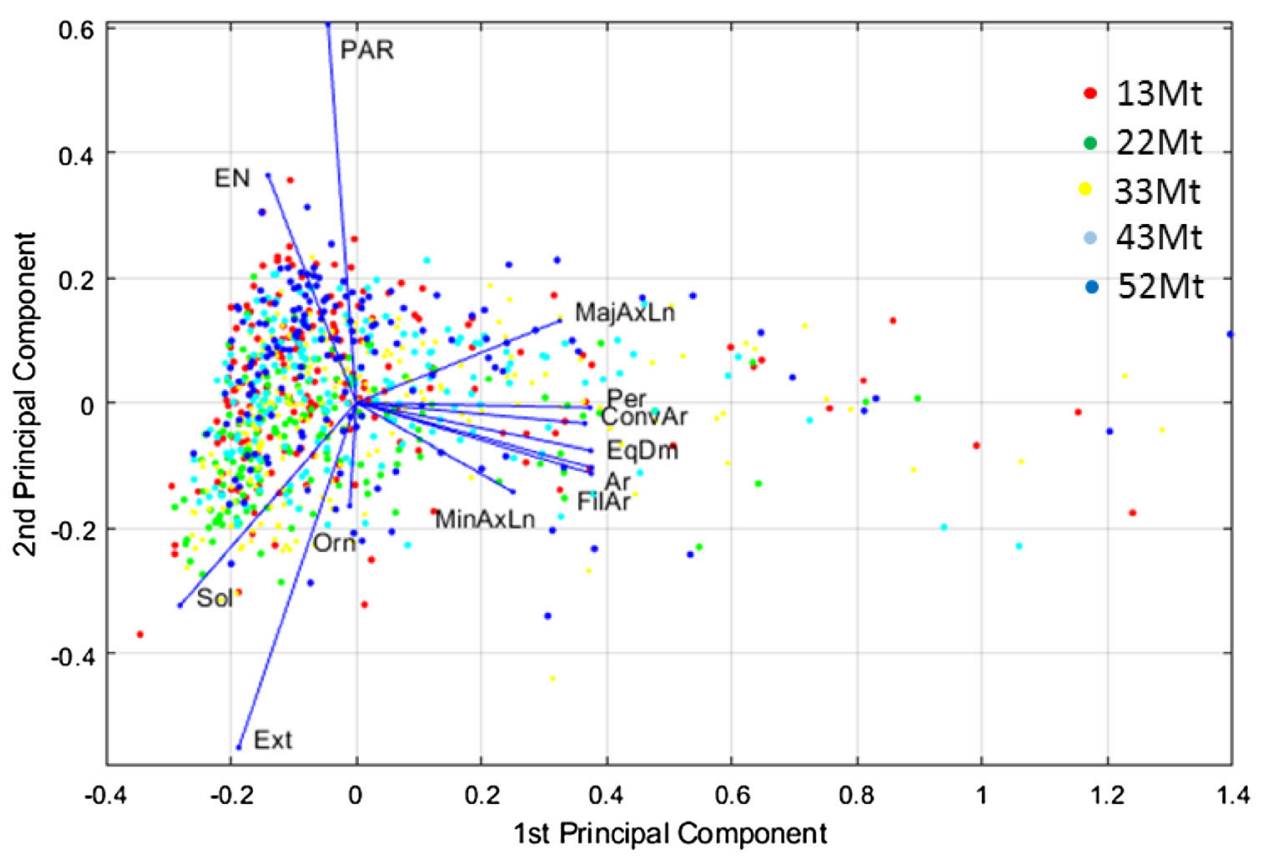

Fig. 12 The features and weights of $P c 1$ and $P c 2$

Fig. 13 The mean $P c 1$ and $P c 2$ values versus the frog's lifetime

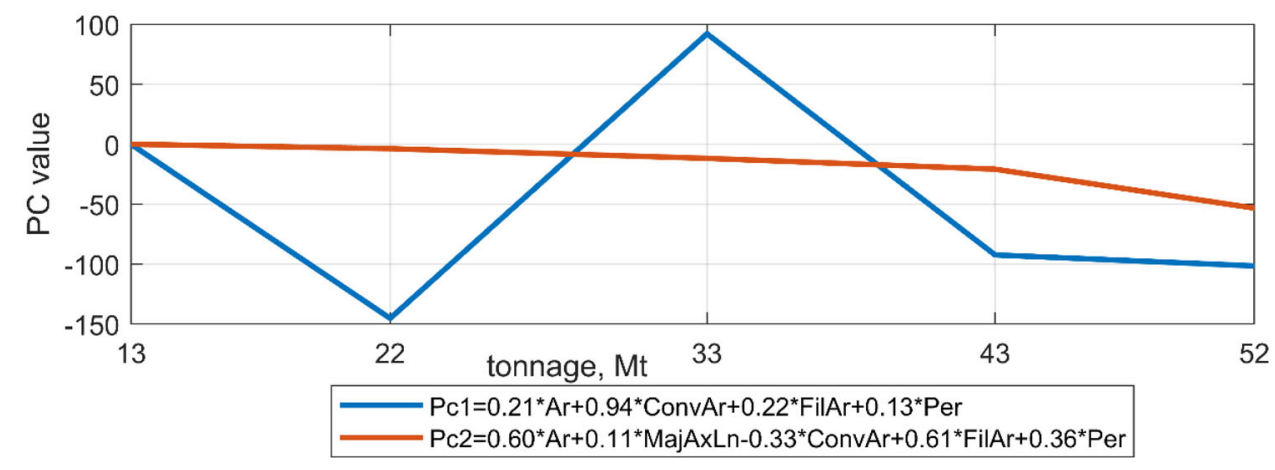

main significant features. The disadvantage is that the PCA is an unsupervised learning approach that does not take into account response variable. Therefore, the relation of the principal components to the crossing lifetime is determined after the analysis. Other supervised linear techniques, like partial least square regression or lasso regularisation [24, 25], could provide additional improvements.

\section{Validation of the Method}

The discovered relation of $P c 2$ to the tonnage only indicates some systematic changes in the form of MPI cracks during the crossing's lifetime. It should be verified if the changes also have a relation to the faults observed on the rolling surface. The validation of the developed statistical method is performed using an independent information source-the available HRPI images. The problem of the 
validation using MPI images is that the crack images disappear as soon as the visual fault occurs. Therefore, the last MPI images before the fault occurrence are used for the validation. Figure 14 (above) shows the MPI image with crack ranking according to the $P c 2$ criterion for a lifetime of $43 \mathrm{Mt}$. Cracks that are most likely to cause surface faults are coloured red. Figure 14 (below) shows the HRPI image of the same part of the rolling surface at $52 \mathrm{Mt}$-after the first damages have appeared. Two prominent groups of cracks are found in the Fig. 14 (below): the right group has grown to a surface damage at $52 \mathrm{Mt}$, the left shows an evident initiation of damage. Serious damage could be expected within the next $10 \mathrm{Mt}$.

\section{Prediction of Remaining Useful Life Based on the Inspected Principal Component Value}

To determine the statistically significant relation of $P c 2$ to the lifetime, a regression analysis is carried out. The regression diagram (Fig. 15) depicts the data points in $P c 2$ form, and a polynomial fit with the $95 \%$ function confidence bounds. The HRPI image for 52-Mt inspection shows a frog surface fault (zone highlighted in red in Fig. 15).

To reveal the possibilities of the remaining life prediction, the following considerations are taken into account (Fig. 16). The inspection delivers a principal component (PC) value at some inspection time $B_{R, 0}$. The intersection of the value with confidence bounds delivers $B_{R \text {,min }}$ and $B_{R, \max }$. The estimation error of the remaining useful life can be determined with the three values as well as the fault prediction horizon.
The first indication in crack features on the future crack development can be found already on 33-Mt MPI images. However, due to the prognosis error, a prediction earlier than $38 \mathrm{Mt}$ (Fig. 15) is not possible. The beginning of visual rail surface faults is also difficult to be determined exactly due to relatively infrequent inspections. Therefore, it could be supposed that the significant fault could have appeared approximately in the middle between the two neighbouring inspections at 43 and $52 \mathrm{Mt}$. Therefore, the prediction of the fault is possible within a prediction horizon of not more than $11 \mathrm{Mt}$.

\section{Conclusion and Subsequent Studies}

The investigation of MPI images of the rolling surface on a crossing nose during a frog's lifecycle has shown that they have a significant statistical relation to the frog's lifetime. The relation is however not evident for simple statistical estimation due to the strong influence of random factors. The application of PCA helps to select the meaningful features carrying information about the fatigue state of the rolling surface. The method validation, using new independent data, shows that the method is able to find the cracks that lead to the surface damages. The regression analysis shows that by using surface crack images it is possible to detect the state changes and forecast the rolling surface damages within a prediction horizon of up to $11 \mathrm{Mt}$.

However, the presented method also has some drawbacks that could be considered as problems for subsequent studies. The major practical problem is the application of the time-consuming MPI imaging inspection method for railway infrastructure with a low degree of automatization.

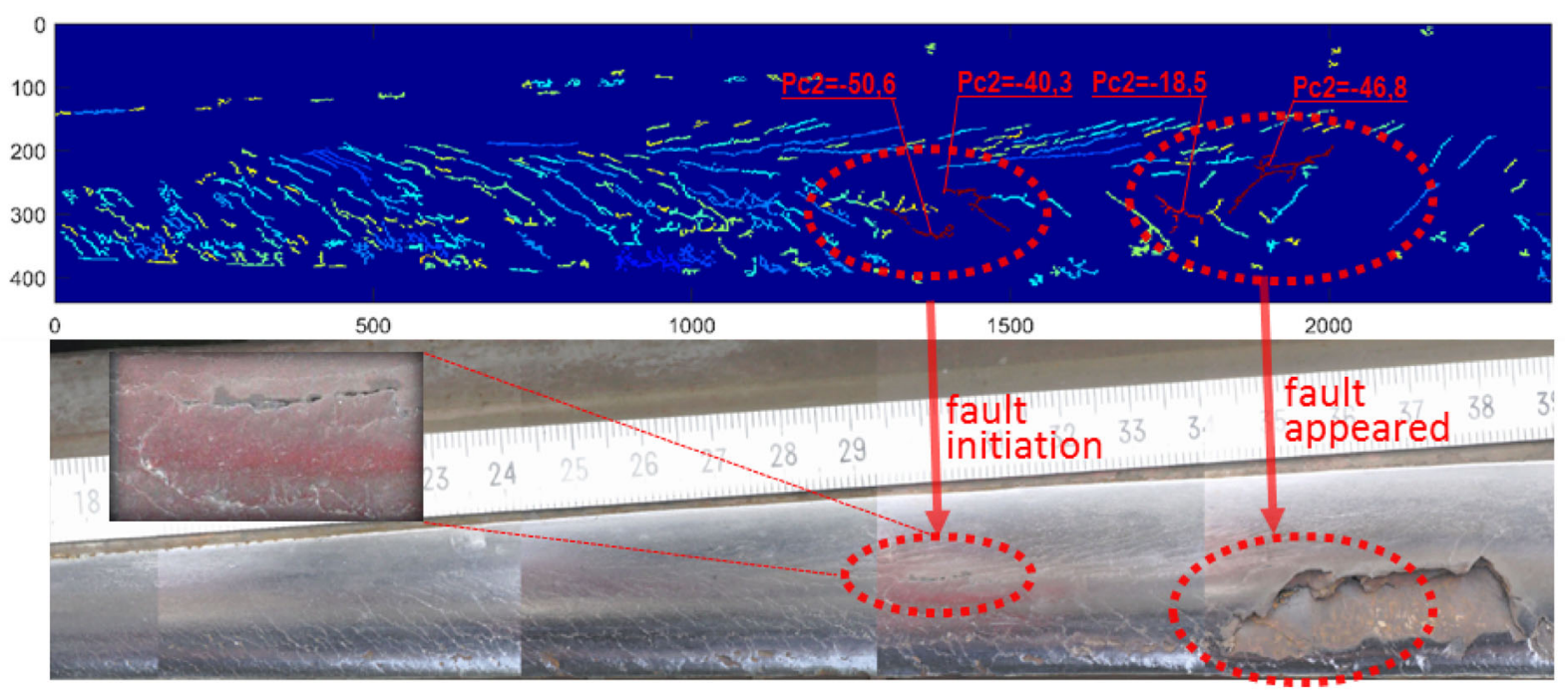

Fig. 14 The predicted faults with MPI imaging at $43 \mathrm{Mt}$ (above) and appeared faults after $52 \mathrm{Mt}$ (below) 
Fig. 15 The regression of $P c 2$ values versus the frog's lifetime

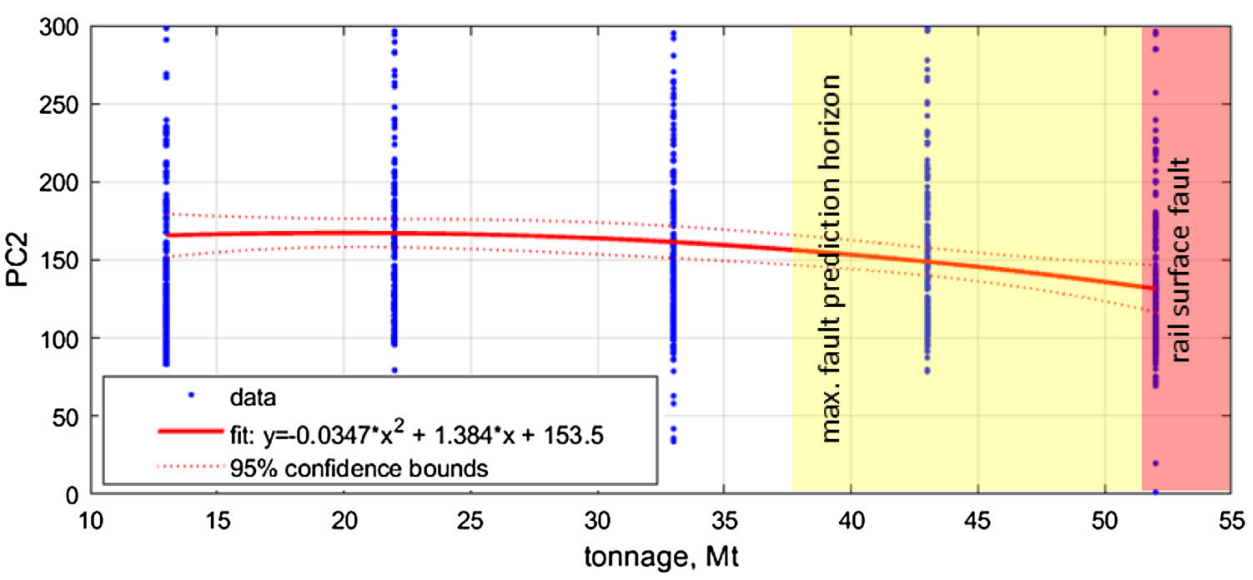

Fig. 16 Assessment of the remaining useful life and prognosis error

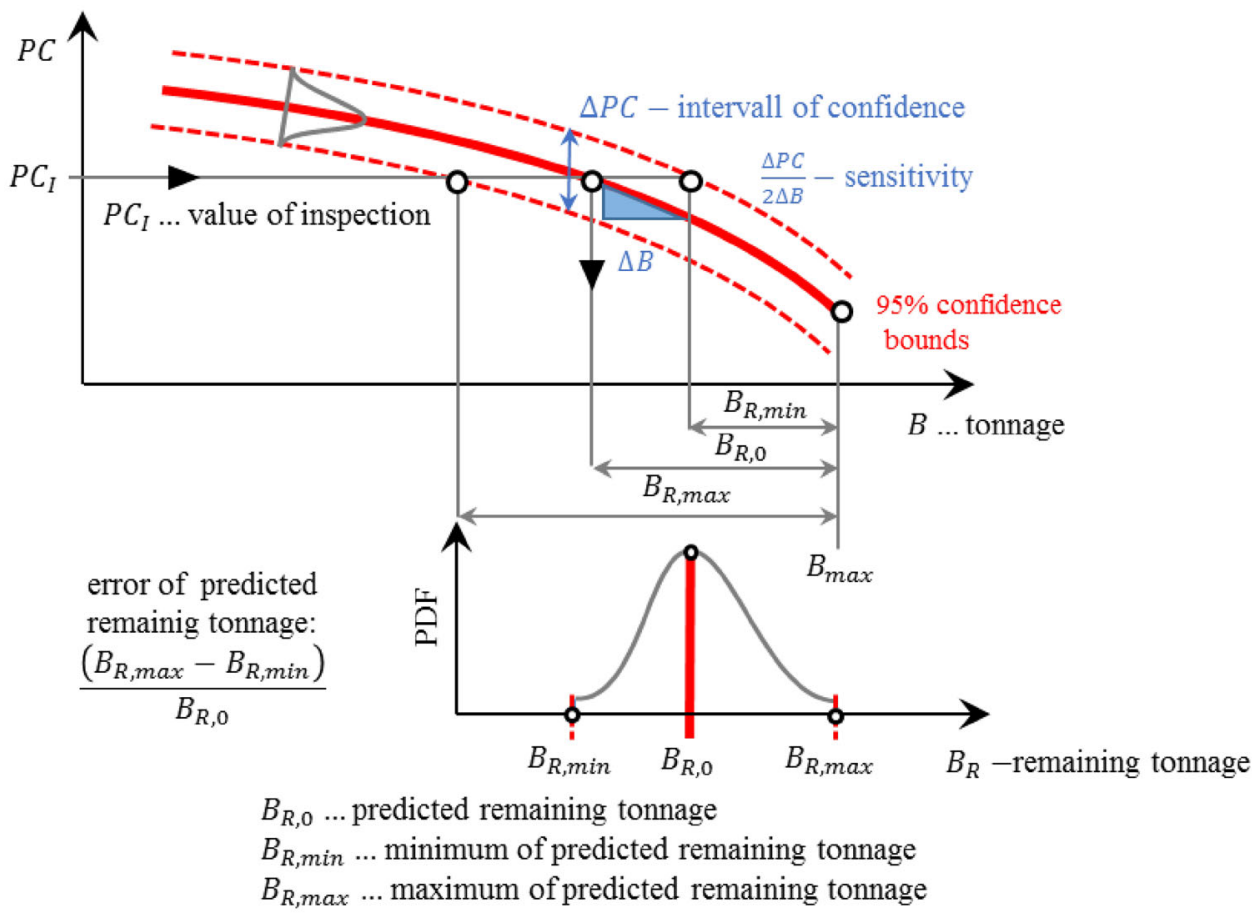

The HRPI inspection method could be used as alternative method, but its application raises the problem of image processing. The problem could be solved using deep learning image processing methods.

Another way to extend the prediction horizon exists in the improvement of statistical information. The main problem here is that the number of cracks that leads to the damages is relative to the general statistics. The problem could be solved in two ways: either by increasing the statistics with new information, or by performing a qualitative improvement of the existing information. New feature measures could be used to describe the internal properties of the crack objects.
Acknowledgements The authors would like to acknowledge the Germany Railway Company (DB Systemtechnik GmbH) and WITT Elektronik $\mathrm{GmbH}$ for their experimental and financial supports.

Open Access This article is distributed under the terms of the Creative Commons Attribution 4.0 International License (http://crea tivecommons.org/licenses/by/4.0/), which permits unrestricted use, distribution, and reproduction in any medium, provided you give appropriate credit to the original author(s) and the source, provide a link to the Creative Commons license, and indicate if changes were made.

\section{Compliance with Ethical Standards}

Conflict of interest The authors declare that they have no conflict of interest. 


\section{References}

1. Fendrich L, Fengler W (2013) Handbuch Eisenbahninfrastruktur (Field manual Railway Infrastructure). Springer, Berlin. https:// doi.org/10.1007/978-3-642-30021-9

2. Zoll A, Gerber U, Fengler W (2016) Das Messsystem ESAH-M (The measuring system ESAH-M). EI-Eisenbahningenieur Kalender 1:49-62. ISSN: 0934-5930

3. Gerber U, Zoll A, Fengler W (2013) Fahrzeugbasierte Beurteilung des Herzstückverschleißes (Vehicle-based assessment of wear on common crossings). EI-Eisenbahningenieur 05:26-30. ISSN: 0013-2810

4. Sysyn M, Kovalchuk V, Jiang D (2019) Performance study of the inertial monitoring method for railway turnouts. Int J Rail Transp 7(2):103-116. https://doi.org/10.1080/23248378.2018.1514282

5. Gerber U, Zoll A, Fengler W (2015) Verschleiß und Fahrflächenermüdung an Weichen mit starrer Herzstückspitze (Wear and Rolling Contact Fatigue on common crossings of railway turnouts). ETR Eisenbahntechnische Rundschau 01:36-41. ISSN: 0013-2845

6. Kovalchuk V, Sysyn M, Hnativ Y, Bal O, Parneta B, Pentsak A (2018) Development of a promising system for diagnosing the frogs of railroad switches using the transverse profile measurement method. East Eur J Enterp Technol 92(2):33-42. https://doi. org/10.15587/1729-4061.2018.125699

7. http://www.graw.com/downloadprod/product-leaflet-scorpion.pdf

8. http://www.witt-solutions.de/assets/Witt_Broschuere_Bahn_a. pdf

9. Kireev A (2018) Increase of informativity of two-frequency method of defectometry at ultrasonic control of details and units of railway rolling stock. Vestn Railw Res Inst 77(3):182-187. https://doi.org/10.21780/2223-9731-2018-77-3-182-187

10. Sein S, Matos JC, Idnurm J (2017) Statistical analysis of reinforced concrete bridges in Estonia. Baltic J Road Bridge Eng 12(4):225-233. https://doi.org/10.3846/bjrbe.2017.28

11. Soghomonyan V, Chibukhchyan G, Chibukhchyan H (2018) Diagnostics of fatigue fractures of building structures elements. Mag Civ Eng 4:195-203. https://doi.org/10.18720/MCE.80.17

12. Sysyn M, Nabochenko O, Kovalchuk V, Gerber U (2019) Evaluation of railway ballast layer consolidation after maintenance works. Acta Polytech 59(1):77-87. https://doi.org/10.14311/ap. 2019.59.0077
13. Storcz T, Ercsey Z, Várady G (2018) Histogram based segmentation of shadowed leaf images. Pollack Period 13(1):22-32. https://doi.org/10.1556/606.2018.13.1.2

14. Hůlková M, Pavelka K, Matoušková E (2018) Automatic classification of point clouds for highway documentation. Acta Polytech 58(3):165-170

15. Shi Y, Cui L, Qi Z, Meng F, Chen Z (2016) Automatic road crack detection using random structured forests. IEEE Trans Intell Transp Syst 17(12):3434-3445. https://doi.org/10.1109/tits.2016. 2552248

16. Bolkeny I, Fuvesi V (2018) Ai based predictive detection system. Pollack Period 13(2):137-146. https://doi.org/10.1556/606.2018. 13.2.14

17. Santur Y, Karaköse M, Akin E (2017) A new rail inspection method based on deep learning using laser cameras. In: IDAP 2017-international artificial intelligence and data processing symposium, 8090245. https://doi.org/10.1109/IDAP.2017. 8090245

18. Hajizadeh S, Núñez A, Tax D (2016) Semi-supervised rail defect detection from imbalanced image Data. IFAC-PapersOnLine 49(3):78-83. https://doi.org/10.1016/j.ifacol.2016.07.014

19. Wang L, Hang Y, Luo S, Luo X, Jiang X (2011) Deblurring gaussian-blur images: a preprocessing for rail head surface defect detection. In: Proceedings of 2011 IEEE international conference on service operations, logistics and informatics, vol 59, pp 451-456. https://doi.org/10.1109/SOLI.2011.5986603

20. Sysyn M, Gruen D, Gerber U, Nabochenko O, Kovalchuk V (2019) Turnout monitoring with vehicle based inertial measurements of operational trains: a machine learning approach. Commun Sci Lett Univ Zilina 21(1):42-48

21. Sysyn M, Gerber U, Gruen D, Nabochenko O, Kovalchuk V (2019) Modelling and vehicle based measurements of ballast settlements under the common crossing. Eur Transp Int J Transp Econ Eng Law 71:1-25

22. Oge $M$ (2011) Practical image and video processing using MATLAB. Wiley-IEEE Press, New York, pp 299-335

23. Kong X, Hu C, Duan Z (2017) Principal component analysis networks and algorithms. Springer, Singapore. https://doi.org/10. 1007/978-981-10-2915-8

24. Bramer M (2016) Principles of data mining, 3rd edn. Springer, London, pp 1-526. https://doi.org/10.1007/978-1-4471-7307-6

25. James G, Witten D, Hastie T, Tibshirani R (2013) An introduction to statistical learning with applications in R. Springer, New York, pp 373-385 